\title{
Technological Interventions for Autism Spectrum Disorder (ASD)
}

\author{
Nour Al Moussawi \\ Joachim Diederich \\ American University of Beirut \\ Department of Psychology \\ Riad El-Solh / Beirut 11072020 \\ Lebanon
}

\section{Introduction}

While communication is changing all around us, psychological practice has not fundamentally changed for more than a hundred years. Psychologists deliver services by talking to people in an office environment or out in the field. While the nature of psychological assessment and therapy may have changed over the years, the formal setting has not: a professional (the psychologist) and a client generally talk one-on-one or in the presence of others.

However, with the arrival of artificial intelligence (AI) in the workplace, psychological service delivery is set to change dramatically. Mobile phone apps, for instance, can analyse speech and language to detect indicators of depression and provide instant feedback to both psychologists and clients. Although online versions of cognitive-behaviour therapy have been available for more than a decade, what is emerging now are "AI psychologists" - programs that are empowered by vast knowledge bases on mental health and how to solve very human problems. These programs talk to people in ways that are almost indistinguishable to the way that human psychologists do. Importantly, they are available anytime (e.g. on your mobile phone), everywhere. And they are cheap. This is psychological expertise on tap, 24/7.

The focus here is to explore and review the role of technology in psychological interventions, in particular for adults. This paper introduces the current definition of autism spectrum disorder (ASD) and the main psychological intervention: Applied Behavioural Analysis (ABA). This is followed by a review of current technology to enhance psychological practice.

\section{Autism in the DSM}

Autism is a neurodevelopmental disorder that changed with every new edition of the DSM (The Diagnostic and Statistical Manual of Mental Disorders): in DSM-I (1952), it was placed under schizophrenic reaction -childhood type, in DSM-II (1968) it was placed under schizophrenia childhood type, in DSM-III (1980) it was moved to a separate category titled infantile autism, in DSM-III-R (1987) the name changed to autistic disorder and in DSM-IV (1990) and DSM-IV-R (2000), it was characterized as one of the five pervasive developmental disorders (PDDs). Finally, in the newest version DSM-5, it was renamed to become a broad category: Autism Spectrum Disorder (Gensler, 2012). DSM-5 also featured the removal of Asperger's disorder as an independent diagnostic category and its inclusion in ASD which generated significant debate

(Parsloe and Babrow, 2016). ASD in DSM-5 has two dimensions, social 
relations/communication and restricted interests/repetitive behavior with seven criteria (Zuddas, 2013).

DSM-5 also incorporated a severity scale for the assessment of autism that could facilitate determination of therapy options and prognosis (Mehling and Tasse, 2016). Severity was assessed in previous DSM versions according to the level of intellectual functioning and ASD symptoms but it was shown to fall short of capturing properly the diversity among people diagnosed with ASD (Di Rezze et al., 2012; in Mehling and Tasse (2016) p.2001). The new scale consists of three severity levels: from assistance to very substantial support (Kite, Gullifer and Tyson, 2013). According to Mandy et al. (2012), the scale was necessary because a single measure in line with previous criteria (IQ and symptomology) could not correlate well with the new two-dimensional diagnosis of ASD (Mandy et al., 2012; in Mehling and Tasse (2016) p.2010). For instance, a measure of 10 on the old scale could be attributed entirely to social communication deficits in one individual and to repetitive behaviour in another, so two individuals with the same severity level can have a different prognosis (Hus et al., 2014; in Mehling and Tasse (2016) p.2010). The severity level in DSM-5 is assessed using clinical interviews but standardized measures of severity (ADOS-II, SRS-2, Vineland-2 for social communication and RBS and ADOS II for repetitive and restricted behaviors) can be useful in informing the decision making process (Mehling and Tasse, 2016).

Concerns were expressed about the removal of Asperger's from DSM-5 (Zuddas, 2013). One of these concerns is that some individuals will no longer meet the criteria for diagnosis and will lose public health support services (Zuddas, 2013). However, rates of diagnosis are increasing even though it is unknown whether the increase is due to an elevated awareness, the broader diagnostic criteria or a true increase in the incidence of ASD (Kulage, Smaldone and Cohn, 2014). Another concern expressed during the transition to DSM-5 was that autism has more negative connotations than Asperger's which may lead to increased stigma (Kite, Gullifer and Tyson, 2013). However, at least one study has shown that there is no support for this claim and that both labels are advantageous for help-seeking and optimism about treatment success (Ohan, Ellefson and Corrigan, 2015).

\section{Applied Behavioral Analysis}

Autism Spectrum Disorder is a neurodevelopmental disorder that is characterized by deficits in social interaction and communication as well as a pattern of restricted interests and repetitive behaviors (American Psychiatric Association, 2013). The objective here is to offer a closer look at $\mathrm{ABA}$ as an intervention for individuals with ASD. In particular, we focus on technological approaches to ABA, thereby taking the intervention to a new level.

B.F Skinner is a principal figure in the field of behaviorism and is known for his fundamental contributions to behaviour therapy (Labrador, 2004). His operant model was an essential contribution to this field and includes a three-part contingency (the ABC): An antecedent (an event that triggers behaviour), a reaction (response or no response) and the consequence that follows the behaviour (Donaldson and Stahmer, 2014). This model is based on the premise that learning is the result of the consequence following the behavior which can either strengthen, modify or weaken it and thus affecting the probability of its reoccurrence. This operant model is 
the foundation of Applied Behavior Analysis (ABA) which is a scientific approach to examining behavior (Donaldson and Stahmer, 2014). ABA has significant empirical support for treating children with autism (Donaldson and Stahmer, 2014). According to the Center for Disease Control and Prevention (CDC, 2014), there is a continuing rise in the prevalence of autism, currently around 1 in 68 children (Donaldson and Stahmer, 2014).

Ivar Lovaas sought to improve the lives of children with autism; he was the pioneer of early ABA interventions and influenced their availability in the 1970s (Smith and Eikeseth, 2011). ABA relies on the operant model to teach new skills, maintain the desired behavior, generalize it and reduce unwanted responses. ABA includes two parts: assessment and intervention (Steege, Mace, Perry and Longenecker, 2007). In the assessment phase, behavior is measured and the interactions between certain patterns of behavior and the environment are identified. Assessment is done before, during and after the intervention to choose the appropriate treatment plan, monitor change and introduce modifications to the intervention if necessary (Steege et al., 2007). During the intervention stage, different techniques are employed including discrete trial training, pivotal response training, prompting and prompt fading among others (Dillenburger and Keenan, 2009).

Discrete trial training (DTT) breaks down tasks into simpler components that are taught one by one (Steege et al., 2007). It involves four stages: (1) presentation of the discriminative stimulus, (2) performance of the targeted response or an approximation of it, (3) reinforcement that is not directly related to the behavior such as a sticker and (4) intertrial interval and recording of data. Pivotal response training (PRT) is another ABA approach using a reinforcer the child likes and is related to the behavior. It also employs the antecedent, behavior and consequence model. DTT has been shown to be effective in teaching skills within a single unit of behavior as opposed to sequential behavior (Steege et al., 2007).

DTT in comparison to PRT in the field of language and generalization was studied by Mohammadzaheri, Koegel, Rezaee and Rafiee (2014). The study is a randomized clinical trial of 18 boys and 12 girls of ages between 6 and 11 diagnosed with ASD according to the DSM-IVTR diagnostic criteria. Following baseline language assessment for the children, individuals were assigned to either the DTT or PRT group and were matched according to age, sex and MLU (mean length of utterance). The DTT group had items and activities chosen by the teacher, targets tasks were worked on exclusively, reinforcers were unrelated to the task (example: food) and were provided for correct behavior or successive approximation of the behavior. In the PRT group, the material was chosen by the child, target tasks were interspersed with already mastered tasks, reinforcers were related to the target behavior and they were provided after all attempts (Mohammadzaheri et al., 2014). The results of the study were that PRT is more effective in improving social communication skills. This could be explained by the increased interest of children in the sessions due to choosing items and activities they are interested in, greater generalization in the PRT group and a more widespread effect due to focus on pivotal behaviors not on individual target ones (Mohammadzaheri et al., 2014).

Individuals with autism have difficulties communicating and performing some tasks, which causes problems in social contexts, so the need for developing proper communication and adaptive skills increases in adulthood to ensure quality of life (Fitzpatrick, Minshew and Eack, 
2013). Psychosocial interventions are useful in helping individuals with ASD and the effect size of such interventions is positive ranging between 0.14 and 3.59 (Fitzpatrick, Minshew and Eack, 2013). Behavioral interventions specifically are shown to be effective in teaching adults with autism a large range of skills (Roth, Gillis and Reed, 2014). A systematic review of behavioral intervention research on adaptive skills in young adults with ASD has shown that interventions with low or high tech assisted procedures such as video modeling, visual cues, reinforcement contingencies and corrective feedback using prompts are effective in assisting adults to develop adaptive life skills (Palmen, Didden and Lang, 2012). Another review of researches on the topic conclude that ABA is highly effective in teaching adaptive skills to individuals of wide range of disabilities including adults with ASD (Matson, Hattier and Belva, 2012).

\section{Technological interventions for children}

Technological support has been promising for individuals with autism, including robots (Fischer, Pereira, Gandomi, Fathima and Fischer, 2017). PABI, a penguin-like humanoid robot, was built to assist in ABA especially in DTT. PABI's eyes have cameras in them, a screen in its body that interacts with the cameras and a motion controller as well as audio and a tablet computer for interaction. This robot can communicate efficiently with the child and assess attention levels, gestures, gaze direction and affect (Fischer et al., 2017). The child interacts with stimuli presented by the robot on the tablet, i.e. PABI shows behaviours that act as prompts and then provides reinforcers or corrections. PABI helps to increase the child's interest and engagement in the sessions and promotes the teacher's attention to certain aspects of the interaction. Hence, the technology supports the teacher as well who is still responsible for implementing the intervention and assessing the performance (Fischer et al., 2017). Another study has shown that children with autism prefer synthetic voices and interact with them better than natural voices and this could be due to differences in the level of prosody such as the melody in the voice and the timing of speech (Herring, Kear, Sheehy and Jones, 2017).

The PECS technique (Picture Exchange Communication System) is known to help children communicate using pictures (Agius and Vance, 2016). The child gives a person a card with the picture of an object or a certain symbol in exchange for the item. Furthermore, PECS teaches the child to communicate with different partners and to discriminate between symbols so that the child can make independent requests (Agius and Vance, 2016). A recent study utilizes this method in combination with instant messaging (IM) to permit users with autism to send a message to other users through a mobile application named the Prospect (Raja, Saringat, Mustapha, and Zainal, 2017). In Prospect, PECS cards are part of the application; each card has a picture with its discription. Then, the user sends the digital card as an SMS to another person as an alternative form of communication (Raja et al., 2017). The application introduces the children to technology and social networks, thereby enhancing their communication skills and provides an amusing interactive learning experience (Raja et al., 2017).

A recent project looked into the effectiveness of "wait cards" on the tolerance of delayed reinforcement in children with autism (Imamoto and Monji, 2016). In training sessions, a 6-year old child was given a wait card whenever he asked for a reinforcer and this wait card could then be exchanged for the reinforcer after a specific time interval. The waiting duration was increased gradually to a maximum of 80 seconds. The technique was then implemented in a restaurant by 
the child's mother. As a result of this technique, the child was able to reduce problem behaviors and spend the waiting time appropriately (Shigeru and Kyoko, 2016).

\section{Technological interventions for adults with ASD}

Palmen, Didden and Arts (2008)employed a non-concurrent multiple baseline design on improving question-asking skills. Nine individuals across three groups between 17 and 25 years participated in the study, all at the high-functioning end of autism with a full-scale IQ higher than 70 on verbal intelligence but with difficulties initiating questions (Palmen, Didden and Arts, 2008). First, during the introduction phase, the trainers explained to the participants the aim of the training and the criteria for a correct question as well as a strategy (on a flowchart to serve as a visual cue). Second, an evaluation of simulated conversations took place whereby the participants were presented a conversation, a topic was introduced, a question was asked and an answer was provided. The trainer then modelled the evaluation of the questions by explaining which were correct and which were not. This was done by playing the audiotape and asking the participants to assess the question by putting a pawn in one of the two boxes (boxes were titled "correct" or "incorrect"). Evaluations were then discussed, feedback was given, and in the case of an incorrect evaluation, the task was repeated. Finally, there was a role-play between each participant and the trainer who initiated a conversation and the participant was given a chance to ask a question and was reinforced by points. The other participants were given the opportunity to evaluate the question of the participant and were also reinforced if they did it correctly. The participant who earned the most points or showed improvement from session to session is then praised (Palmen, Didden and Arts, 2008).

The results of the study showed that the approach resulted in a clinically and statistically significant improvement in the skill of question asking, the improvement was maintained and generalized to anatural setting. Results could also point to he fact that the training strengthened self-control and enhanced self-management skills (Palmen, Didden and Arts, 2008).

Another study investigated the efficacy of power cards in improving conversational skills of adolescents with Asperger's syndrome. The study included the generalization to inclusive classroom settings using a multiple baseline experimental design (Davis, Boon, Cihak and Fore III, 2010). According to Gangon et al. (2003), power cards link the special interest area (SIA) of the child to the desired behavior. Powercards have two components: a power script, i.e. a descriptive script about a problem and a solution along with a picture (Davis et al., 2010). The study included three male participants of high school age diagnosed with ASD and no previous experience with power cards. In addition, there are 20 volunteers without disabilities as conversational partners. A "best hero" who represented the SIA was identified. The power script displayed the hero's attention to other person's interests and the power card included the strategy of the conversation used by the hero. After the participants were introduced to the aim of the cards and the power scripts, they were given the power cards to maintain a conversation focused on other's interests (target behavior). A test was used to ensure the generalization of these conversational skills to other contexts and other partners.

The time participants spent engaged in a conversation focussing on other people's interests was measured and the results showed that this power card strategy improves the conversational skills 
of students. It is helpful in capturing the student's attention by focusing on their area of interest (Davis et al., 2010).

Can video modeling assist in acquiring self-help skills like hand washing (Campbell, Morgan, Barnett and Spreat, 2015)? This is a multiple baseline study including three young adults with ASD and intellectual disability who have the motor skills to appropriately press the play button of a MP4 video player. After assessing the participants, they were presented with the video that demonstrated the task of hand washing step by step. The performance was marked as either "independent" if they initiated a step or "not independent" if they failed to initiate a step or required a prompt to do so. Each participant had a training session once per week for 10 to 15 minutes over four weeks. The results showed that the video modeling technique improved skill acquisition even if the participants did not master the skill, nevertheless they showed progress toward independence (Campbell, et al., 2015).

Some adolescents lack the required fine motor skills or touch sensitivity to press the play button on a portable speech-generating device (SGD). Steven, a 17-year old boy with autism, OCD and ADHD was using an iPod-based SGD device. He was assessed and he exhibited problems activating the speech output function of the iPod. The iPod had pictures of snacks and Steven used it to request an order. If Steven was not able to activate the speech output associated with the request of a snack, he was prompted to repeat it with physical assistance from a trainer. Then, prompting delay time was increased gradually until Steven could initiate the speech output successfully with no need for prompting. The results show that differential reinforcement and delayed prompting were useful in shaping the response topography required to activate this SGD (Kagohara et al., 2010).

Baron-Cohen (1995) and Hobson (1994) showed that emotional regulation and mental state recognition are deficit areas in individuals with ASD (Golan and Baron-Cohen, 2006). Hence the need to develop abilities to recognize emotions and one study investigated the effectiveness of an interactive systematic guide to emotions and mental states: Mind Reading (Golan and BaronCohen, 2006). The emotions and mental states implemented in Mind Reading are properly and systematically organized according to emotional group and developmental levels, and each emotional group is illustrated by a short video that provides clues for recognising these emotions later on. The study was divided into two experiments. First, a controlled trial design using multiple repeated measures was used to compare the effect of using Mind Reading at home with no intervention at all. The following instruments were used: CAM Face-Voice Battery, Reading the Mind in the Eyes task, Reading the Mind in the Voice task and Reading the Mind in Film task. Each test was repeated twice for the treatment and control group (before and after the intervention for the treatment group). The results of the first experiment show improvement in emotional recognition of voices and faces of participants in the treatment group compared to the control group and to their assessment results before the intervention (Golan and Baron-Cohen, 2006).

In the second experiment, three groups were used to compare (1) the effect of using Mind Reading with help from a tutor in a group setting (software and tutor group) to (2) social skills training without the use of Mind Reading (social skills course group) and (3) a general population control group (Golan and Baron-Cohen, 2006). The results show that the software 
and tutor group significantly improved in the emotional recognition of voices more than the control group and in recognition of emotional concept more than the control and social skills group. The improvement was independent of the group or tutor support and it was difficult to generalize the skills to other emotional recognition tasks (Golan and Baron-Cohen, 2006).

Beyond questionnaires, observer-rating scales and clinical interviews, there are hardly any methods that identify mental health conditions that result in an impairment in the recognition of emotional states (Diederich 2016). Such a condition is Alexithymia; the inability to identify and describe emotions. Alexithymia is not a recognized disorder, however, the condition overlaps significantly with Aspergers and autism spectrum disorder. It is also possible that frequently observed psychological problems such as anxiety and depression result in distraction and withdrawal including a lack of awareness of emotional expressions. The Mental State Tracker aims at identifying emotional states and to make this information available for clinical practice.

The Mental State Tracker is a mobile application available for Android, Apple and Windows devices. This mobile app is based on artificial intelligence (AI) techniques and combines a number of features: (1) The recording and analysis of speech to detect a number of emotional states. (2) The transcription of speech and the analysis of the resulting texts by various methods to determine emotional issues. (3) The analysis of transcribed speech includes the determination of suicide risk. (4) Standard questionnaires that are commonly used in clinical psychology and psychiatry are also offered and the results are compared with speech and text analysis.

It is possible to record unrestricted speech of several minutes in length and then to receive a detailed report on the distribution of positive and negative emotion words as well as expressions that indicate excessive self-referral. Current research focusses on chatbots that can be used inside the app to provide psychoeduction once an impairment has been identified.

\section{Conclusion}

In psychology, there is always a need for validating interventions and evaluating their efficacy and effectiveness across various populations. ABA with ASD is a topic that has been receiving interest in the last few decades (Donaldson and Stahmer, 2014). However, there is a significant need for comparison studies of ABA with other approaches such as cognitive therapy supported by technology. Moreover, research is still developing in the field of treating adults with ASD. Future studies could explore the effectiveness of a humanoid-robot in applying ABA DTT techniques to adults. The "wait card" technique could be replicated with adults to check its effectiveness, or a similar technique that teaches tolerance to reinforcers may be developed. Also, the benefits of the Prospect application in improving the communication skills of adults and providing an alternative method of social interaction can be investigated. More attention to interventions with adults is essential and there is a need to fill the gap between research and clinical practice.

\section{References}

Agius, M. M., \& Vance, M. (2016). A comparison of PECS and iPad to teach requesting to preschoolers with autistic spectrum disorders. AAC: Augmentative And Alternative Communication, 32(1), 58-68. doi:10.3109/07434618.2015.1108363 
American Psychiatric Association, 2013. Diagnostic and Statistical Manual of Mental Disorders (5th ed., text revision): DSM-5. Author, Washington, DC.

Bishop-Fitzpatrick, L., Minshew, N. J., \& Eack, S. M. (2013). A systematic review of psychosocial interventions for adults with autism spectrum disorders. Journal Of Autism And Developmental Disorders, 43(3), 687-694. doi:10.1007/s10803-012-1615-8

Campbell, J. E., Morgan, M., Barnett, V., \& Spreat, S. (2015). Handheld devices and video modeling to enhance the learning of self-help skills in adolescents with autism spectrum disorder. OTJR: Occupation, Participation And Health, 35(2), 95-100. doi:10.1177/1539449215570040

Davis, K. M., Boon, R. T., Cihak, D. F., \& Fore, C. I. (2010). Power cards to improve conversational skills in adolescents with Asperger syndrome. Focus On Autism And Other Developmental Disabilities, 25(1), 12-22. doi:10.1177/1088357609354299

Dickstein-Fischer, L. A., Pereira, R. H., Gandomi, K. Y., Fathima, A. T., \& Fischer, G. S. (2017). Interactive tracking for robot-assisted autism therapy. Paper presented at the $A C M / I E E E$ International Conference on Human-Robot Interaction, 107-108. doi:10.1145/3029798.3038390

Diederich, J., The Mental State Tracker - Language Anlysis for Mental Health. The Australian \& New Zealand Mental Health Association, January 2017.

Dillenburger, K., \& Keenan, M. (2009). None of the As in ABA stand for autism: Dispelling the myths. Journal Of Intellectual And Developmental Disability, 34(2), 193-195. doi:10.1080/13668250902845244

Donaldson, A. L., \& Stahmer, A. C. (2014). Team collaboration: The use of behavior principles for serving students with ASD. Language, Speech, And Hearing Services In Schools, 45(4), 261-276. doi:10.1044/2014_LSHSS-14-0038

Gensler, D. (2012). Autism spectrum disorder in DSM-V: Differential diagnosis and boundary conditions. Journal Of Infant, Child \& Adolescent Psychotherapy, 11(2), 86-95. doi:10.1080/15289168.2012.676339

Golan, O., \& Baron-Cohen, S. (2006). Systemizing empathy: Teaching adults with Asperger syndrome or high-functioning autism to recognize complex emotions using interactive multimedia. Development And Psychopathology, 18(2), 591-617. doi:10.1017/S0954579406060305

Herring, P., Kear, K., Sheehy, K., \& Jones, R. (2017). A virtual tutor for children with autism. Journal of Enabling Technologies, 11(1), 19-27. doi:10.1108/JET-01-2016-0006

Imamoto, S., \& Monji, K. (2016). Effects of a 'wait card' to teach tolerance of delayed presentation of reinforcers: Child with autism spectrum disorder. Japanese Journal Of Special Education, 54(2), 111-120.

Kagohara, D. M., van der Meer, L., Achmadi, D., Green, V. A., O'Reilly, M. F., Mulloy, A., \& ... Sigafoos, J. (2010). Behavioral intervention promotes successful use of an iPod-based communication device by an adolescent with autism. Clinical Case Studies, 9(5), 328338. doi:10.1177/1534650110379633

Kite, D. M., Gullifer, J., \& Tyson, G. A. (2013). Views on the diagnostic labels of autism and Asperger's disorder and the proposed changes in the DSM. Journal Of Autism And Developmental Disorders, 43(7), 1692-1700. doi:10.1007/s10803-012-1718-2

Kulage, K. M., Smaldone, A. M., \& Cohn, E. G. (2014). How will DSM-5 affect autism diagnosis? A systematic literature review and meta-analysis. Journal Of Autism And 
Developmental Disorders, 44(8), 1918-1932. doi:10.1007/s10803-014-2065-2

Labrador, F. J. (2004). Skinner and the rise of behavior modification and behavior therapy. Spanish Journal of Psychology, 7(2), 178-187. Retrieved from www.scopus.com

Matson, J. L., Hattier, M. A., \& Belva, B. (2012). Treating adaptive living skills of persons with autism using applied behavior analysis: A review. Research In Autism Spectrum Disorders, 6(1), 271-276. doi:10.1016/j.rasd.2011.05.008

Mehling, M. H., \& Tassé, M. J. (2016). Severity of autism spectrum disorders: Current conceptualization, and transition to DSM-5. Journal Of Autism And Developmental Disorders, 46(6), 2000-2016. doi:10.1007/s10803-016-2731-7

Mohammadzaheri, F., Koegel, L. K., Rezaee, M., \& Rafiee, S. M. (2014). A randomized clinical trial comparison between pivotal response treatment (PRT) and structured applied behavior analysis (ABA) intervention for children with autism. Journal Of Autism And Developmental Disorders, 44(11), 2769-2777. doi:10.1007/s10803-014-2137-3

Ohan, J. L., Ellefson, S. E., \& Corrigan, P. W. (2015). Brief report: The impact of changing from DSM-IV 'Asperger's' to DSM-5 'autistic spectrum disorder' diagnostic labels on stigma and treatment attitudes. Journal Of Autism And Developmental Disorders, 45(10), 33843389. doi:10.1007/s10803-015-2485-7

Palmen, A., Didden, R., \& Arts, M. (2008). Improving question asking in high-functioning adolescents with autism spectrum disorders: Effectiveness of small-group training. Autism, 12(1), 83-98. doi:10.1177/1362361307085265

Palmen, A., Didden, R., \& Lang, R. (2012). A systematic review of behavioral intervention research on adaptive skill building in high-functioning young adults with autism spectrum disorder. Research In Autism Spectrum Disorders, 6(2), 602-617. doi:10.1016/j.rasd.2011.10.001

Parsloe, S. M., \& Babrow, A. S. (2016). Removal of Asperger's syndrome from the DSM V: Community response to uncertainty. Health Communication, 31(4), 485-494. doi:10.1080/10410236.2014.968828

Raja, P., Saringat, M. Z., Mustapha, A., \& Zainal, A. (2017). Prospect: A picture exchange communication system (PECS)-based instant messaging application for autism spectrum condition. Paper presented at the IOP Conference Series: Materials Science and Engineering, , 226(1) doi:10.1088/1757-899X/226/1/012088

Roth, M. E., Gillis, J. M., \& DiGennaro Reed, F. D. (2014). A meta-analysis of behavioral interventions for adolescents and adults with autism spectrum disorders. Journal of Behavioral Education, 23(2), 258-286. doi:10.1007/s10864-013-9189-x

Smith, T., \& Eikeseth, S. (2011). O. Ivar Lovaas: Pioneer of applied behavior analysis and intervention for children with autism. Journal Of Autism And Developmental Disorders, 41(3), 375-378. doi:10.1007/s10803-010-1162-0

Steege, M. W., Mace, F. C., Perry, L., \& Longenecker, H. (2007). Applied behavior analysis: Beyond discrete trial teaching. Psychology In The Schools, 44(1), 91-99. doi:10.1002/pits.20208

Zuddas, A. (2013). Autism assessment tools in the transition from DSM-IV to DSM-5. European Child \& Adolescent Psychiatry, 22(6), 325-327. doi:10.1007/s00787-013-0424-8 